\title{
Commentary: Nighttime stars: Intensivist coverage and cardiac surgical outcomes
}

Thomas S. Metkus, MD, ${ }^{\mathrm{a}}$ and Glenn J. R. Whitman, $\mathrm{MD}^{\mathrm{b}}$

\footnotetext{
From the ${ }^{\mathrm{a} D i v i s i o n}$ of Cardiology, Department of Medicine, and ${ }^{\mathrm{b}}$ Division of Cardiac Surgery, Department of Surgery, The Johns Hopkins Medical Institutions, Baltimore, Md.

Disclosures: Authors have nothing to disclose with regard to commercial support.

Received for publication April 26, 2019; accepted for publication April 26, 2019; available ahead of print June 27, 2019.

Address for reprints: Glenn J. R. Whitman, MD, Division of Cardiac Surgery, Department of Surgery, The Johns Hopkins Hospital, Sheikh Zayed Tower, 1800 Orleans St, Ste 7107, Baltimore, MD 21287 (E-mail: gwhitman@jhmi.edu).

J Thorac Cardiovasc Surg 2020;159:1376-7

0022-5223/\$36.00

Copyright (C) 2019 by The American Association for Thoracic Surgery

https://doi.org/10.1016/j.jtcvs.2019.04.078
}

The best strategy to care for critically ill patients overnight inspires great debate. ${ }^{1,2}$ Therefore, Huard and colleagues ${ }^{3}$ should be commended for their work assessing the association of 24-hour intensivist coverage in a cardiac surgery intensive care unit (ICU) with outcomes. They compared 2 eras in their cardiac surgery ICU. During time period A, board-certified intensivists provided care during daytime hours and a resident physician or fellow provided care off-hours with backup by the on-call cardiac surgeon. During time period B, 2 intensivists provided care during the day and 1 intensivist provided care overnight. The intensivist group was multidisciplinary, including critical care-trained anesthesiologists, pulmonologists, internists, surgeons, cardiologists, and nephrologists. Furthermore, the group personnel remained stable without physician turnover through 8 years across both eras. Using propensity score matching, the authors demonstrate that 24-hour intensivist coverage was associated with lower mortality, lower rates of cardiac arrest, and shorter duration of mechanical ventilation. Although ICU use was higher with higher rates of ICU readmissions, mortality among patients readmitted to ICU during era B was lower. Mortality associated with postoperative complications, commonly referred to as failure to rescue ${ }^{4}$ decreased during era $\mathrm{B}$ in every instance. The improved mortality experienced was seen in only the higher-risk patients with a European system of cardiac risk evaluation II score $>5 \%$.

A possible interpretation of these findings, which are broadly consistent with a similarly structured, retrospective Canadian analysis by Benoit and colleagues, ${ }^{5}$ is that 24hour intensivist coverage improves outcomes due to expert critical care. Although these results contrast with the sole, randomized trial of nighttime intensivist staffing that did not demonstrate reduced mortality, ${ }^{6}$ that study was conducted in a single, academic medical ICU and may not generalizable to this population. An alternate explanation for the findings of Huard and colleagues ${ }^{3}$ is that the observed 8-year time period.

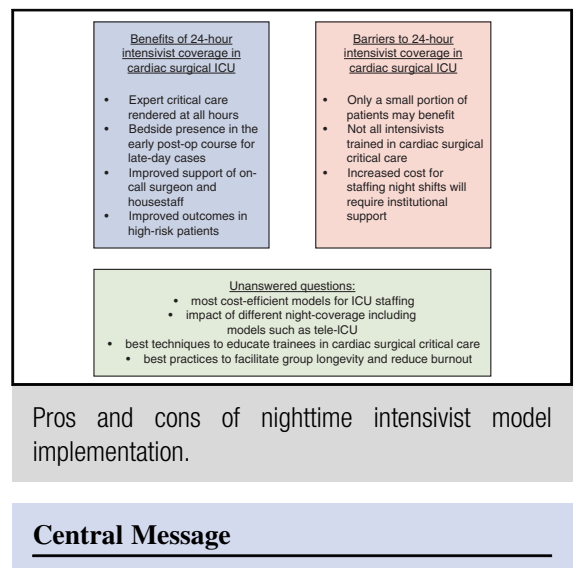

Nighttime coverage by intensivists providing cardiac surgical care improved multiple metrics, but decreased mortality only in high-risk patients. Implementation of the model requires resources and possible modifications.

See Article page 1363.

improvement was due to transitioning nighttime care from less-experienced resident physicians and fellows to more experienced clinicians. Finally, as in any study comparing two eras using propensity score methods, unmeasured confounders certainly exist and could account for a portion of the observed effect. To allay this concern, the authors performed a robust set of sensitivity analyses and describe that most institutional factors remained constant over the

What broad lessons can we learn from these data? First, excellent care is not contingent on critical care training from any single specialty. Similar to our group at Hopkins, the authors' ICU group is multidisciplinary, with representatives from medicine, surgery, anesthesiology, and medical subspecialties. Second, the authors' results occurred in an environment of consistent ICU protocols, nursing ratios, and a stable surgical and intensivist group, all of which could be necessary to realize these benefits. Finally, nighttime ICU coverage benefits high-risk patients the most. But, there are barriers to implementation of 24-hour intensivist coverage. For example, transitioning to this model would require the cost of an additional 4400 hours of ICU intensivist coverage in our center. For hospitals with low volumes and few high-risk patients, this would be prohibitive. Centralizing ICU care for the highest-risk cardiac surgery patients, already described for critical 
care cardiology, ${ }^{7}$ might be an efficient way to realize the benefits gained by full-time intensivist staffing.

On the basis of these data, intensivist stars do come out at night, and they improve cardiac surgery outcomes.

\section{References}

1. Kerlin MP, Halpern SD. Nighttime physician staffing improves patient outcomes: no. Intensive Care Med. 2016;42:1469-71.

2. Wallace DJ. Nighttime physician staffing improves patient outcomes: yes. Intensive Care Med. 2016;42:1467-8.

3. Huard P, Kalavrouziotis D, Lipes J, Simon M, Tardif M-A, Blackburn S, et al. Does the full-time presence of an intensivist lead to better outcomes in the cardiac surgical intensive care unit? J Thorac Cardiovasc Surg. 2020;159:1363-75.e7.
4. Gonzalez AA, Dimick JB, Birkmeyer JD, Ghaferi AA. Understanding the volumeoutcome effect in cardiovascular surgery: the role of failure to rescue. JAMA Surg. 2014;149:119-23.

5. Benoit MA, Bagshaw SM, Norris CM, Zibdawi M, Chin WD, Ross DB, et al. Postoperative complications and outcomes associated with a transition to 24/7 intensivist management of cardiac surgery patients. Crit Care Med. 2017;45: 993-1000.

6. Kerlin MP, Small DS, Cooney E, Fuchs, Bellini LM, Mikkelsen ME, et al. A randomized trial of nighttime physician staffing in an intensive care unit. $N$ Engl $J$ Med. 2013;368:2201-9.

7. Morrow DA, Fang JC, Fintel DJ, Granger CB, Katz JN, Kushner FG, et al. Evolution of critical care cardiology: transformation of the cardiovascular intensive care unit and the emerging need for new medical staffing and training models: a scientific statement from the American Heart Association. Circulation. 2012;126: 1408-28. 